\title{
Synthesis of camphor-derived chiral auxiliaries and their application in asymmetric Morita-Baylis-Hillman reactions
}

\author{
Lulama P. Mciteka, Kevin A. Lobb, and Perry T. Kaye* \\ Department of Chemistry, Rhodes University, Grahamstown 6140, South Africa \\ E-mail: P.Kaye@ru.ac.za
}

DOI: http://dx.doi.org/10.3998/ark.5550190.0017.511

\begin{abstract}
$N$-Substituted 2-exo-hydroxybornyl-10-sulfonamides, prepared as potential chiral auxiliaries for use in asymmetric Morita-Baylis-Hillman $(\mathrm{MBH})$ reactions, have been treated with acryloyl chloride to afford the corresponding 2-exo-acrylate esters as MBH substrates. Reaction of selected 2-exo-acrylate ester substrates with pyridine-4-carbaldehyde and 6-methylpyridine-2carbaldehyde in the presence of DABCO gave the expected $\mathrm{MBH}$ adducts in $>91 \%$ yield and with diastereoselectivities of $7-33 \%$ d.e.
\end{abstract}

Keywords: Camphor, chiral auxiliaries, bornyl acrylates, Morita-Baylis-Hillman reactions

\section{Introduction}

The conformational rigidity and steric demands of the bicyclic camphor system account for its use in the construction of a wide range of chiral auxiliaries. ${ }^{1}$ These include Oppolzer's classic camphorsultams ${ }^{2}$ and the sterically hindered systems developed by Helmchen. ${ }^{3}$ In earlier studies, we have explored the application of camphor-derived chiral auxiliaries in the asymmetric $\alpha$ benzylation of carboxylic esters prepared using the borneol derivative $1,{ }^{4}$ Simmons-Smith asymmetric cyclopropanation of $\alpha, \beta$-unsaturated acetals prepared using the chiral diol $2,{ }^{5}$ and preliminary asymmetric Morita-Baylis-Hillman (MBH) reactions involving chiral acrylate esters. ${ }^{6}$ The chiral auxiliary used in these preliminary $\mathrm{MBH}$ reactions was the $\mathrm{N}$-adamantyl-2-exohydroxybornyl-10-sulfonamide (3), and the promising level of diastereoselectivity observed in one of these reactions prompted us to investigate the preparation of a series of $N$-substituted analogues as potential chiral auxiliaries for asymmetric MBH reactions.

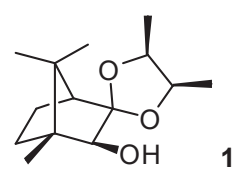

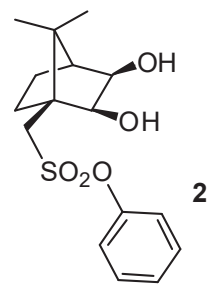

Page 151

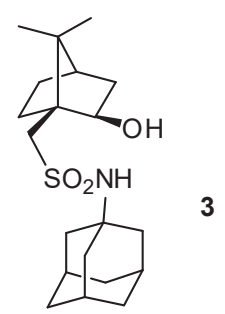

${ }^{\circ}$ ARKAT-USA, Inc. 


\section{Results and Discussion}

Synthetic access to the series of chiral auxiliaries $\mathbf{7}$ and $\mathbf{8}$ and the corresponding acrylate ester derivatives $\mathbf{9}$ and $\mathbf{1 0}$ is outlined in Scheme 1. Thus, each of primary or secondary amines $\mathbf{5 a - j}$ was treated with $(1 S)-(+)$-camphor-10-sulfonyl chloride (4) in the presence of a catalytic quantity of 4(dimethylamino)pyridine (DMAP) in acetonitrile. Work-up and purification furnished the corresponding $N$-substituted camphor-10-sulfonamides 6a-j in good to excellent yields (83-100\%; Table 1). Reduction of the carbonyl group in each of the $N$-substituted camphor-10-sulfonamides 6a-j was effected using $\mathrm{NaBH}_{4}$ to afford the epimeric 2-hydroxybornyl-10-sulfonamides 7a-j and $\mathbf{8 a}-\mathbf{h}, \mathbf{j}$ in moderate to excellent yields $(53-100 \%)$ and, generally, with high diastereoselectivity (100\% in the case of the 2-imidazolyl derivative $7 \mathbf{i}$ ), as shown in Table 1 . The dicyclohexyl derivative $\mathbf{7} \mathbf{j}$ has been used previously as a chiral auxiliary by Oppolzer et al. in asymmetric DielsAlder reactions ${ }^{7}$ and in the asymmetric synthesis of aldols, ${ }^{8}$ halohydrins and epoxides, ${ }^{9}$ and $\alpha$ amino acids. ${ }^{10}$ Semi-preparative HPLC permitted isolation of analytical samples of the 2-exo-and 2-endo-hydroxy epimers, eight of which are new compounds. The C-2 configurations assigned to these epimeric products are supported by their ${ }^{1} \mathrm{H}$ NMR chemical shift and coupling constant data. Dominance of the 2-exo-hydroxy epimers 7 may be attributed to preferential hydride delivery to the less hindered endo face of the carbonyl group in the ketone precursors 6.

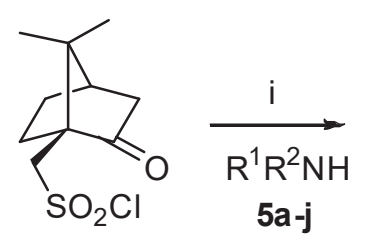

4

\begin{tabular}{l|ll} 
& $\mathrm{R}^{1}$ & $\mathrm{R}^{2}$ \\
\cline { 2 - 3 } $\mathbf{a}$ & benzyl & $\mathrm{H}$ \\
$\mathbf{b}$ & 3-chlorophenyl & $\mathrm{H}$ \\
$\mathbf{c}$ & phenyl & $\mathrm{H}$ \\
$\mathbf{d}$ & furfuryl & $\mathrm{H}$ \\
$\mathbf{e}$ & 2-pyridyl & $\mathrm{H}$ \\
$\mathbf{f}$ & 1-adamantyl & $\mathrm{H}$ \\
$\mathbf{g}$ & 2-picolyl & $\mathrm{H}$ \\
$\mathbf{h}$ & --butyl & $\mathrm{H}$ \\
$\mathbf{i}$ & 2-imidazolyl & $\mathrm{H}$ \\
$\mathbf{j}$ & cyclohexyl & cyclohexyl
\end{tabular}

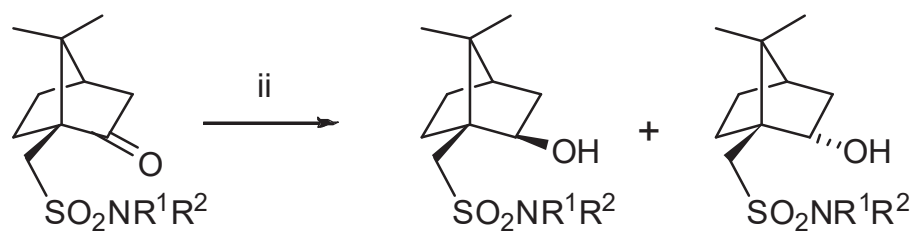

$6 a-j$
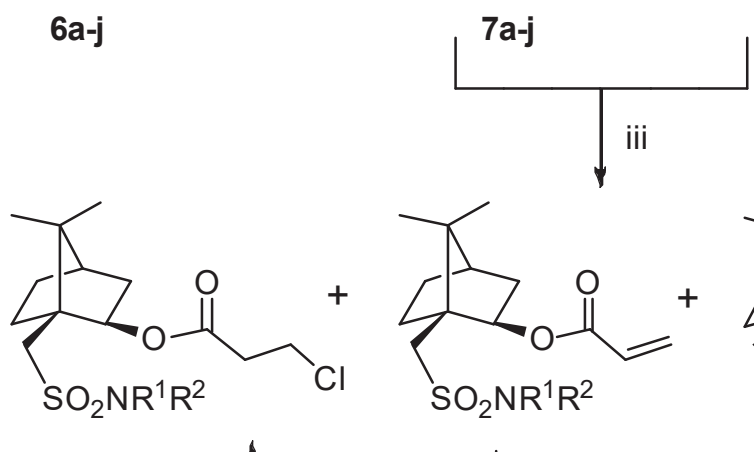

$11 a-c, e, f, j$

9a-c,e-,j
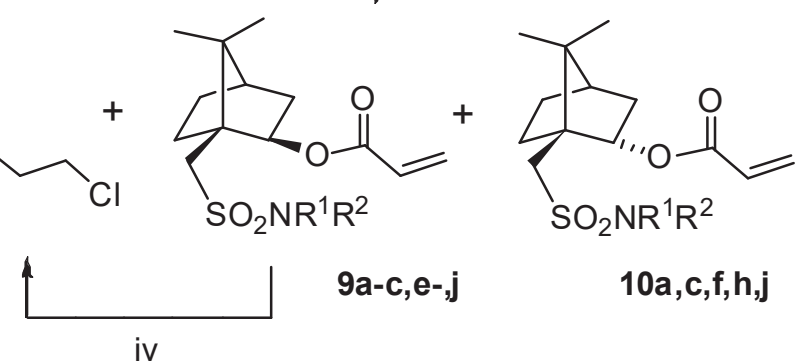

$10 a, c, f, h, j$

Scheme 1. Reagents and conditions: i) DMAP, $\mathrm{CH}_{3} \mathrm{CN}, 0{ }^{\circ} \mathrm{C}$; ii) $\mathrm{NaBH}_{4}, \mathrm{EtOH}-\mathrm{H}_{2} \mathrm{O}, 0{ }^{\circ} \mathrm{C}$; iii) $\mathrm{Al}_{2} \mathrm{O}_{3}, \mathrm{CH}_{2}=\mathrm{CHCOCl}$; and iv) in situ $\mathrm{HCl}$. 
Formation of the acrylate esters $\mathbf{9}$ and $\mathbf{1 0}$ was achieved as reported previously. ${ }^{6}$ The alcohols 7 and $\mathbf{8}$ were added, generally as epimeric mixtures, to neutral $\mathrm{Al}_{2} \mathrm{O}_{3}$ (1.5 eq.), followed by acryloyl chloride (2 eq.). The resulting dispersions were allowed to stand at r.t. for $72 \mathrm{~h}$ without stirring. Flash chromatographic separation of the products, following work-up, proved impossible, necessitating the use of semi-preparative HPLC to obtain analytical samples; even then, the products, in some cases, remained slightly contaminated. The major products were, typically, the 2-exo-acryloyloxybornane-10-sulfonamides 9 and their 2-exo-[(3-chloropropanoyl)oxy]bornane10-sulfonamide derivatives 11, the latter arising from conjugate addition to the former by the $\mathrm{HCl}$ released as a by-product. [We have shown previously ${ }^{6}$ that dehydrochlorination $(\mathbf{1 1} \rightarrow \mathbf{9})$ can be readily achieved using triethylamine.] Minor products, isolated in some cases, included the 2-endo[(3-chloropropanoyl)oxy]bornane-10-sulfonamide derivatives due to the presence of low concentrations of the 2-endo alcohols in the substrate mixtures.

Table 1. Data for the preparation of the camphor-10-sulfonamides 6 and the diastereomeric bornyl alcohols $\mathbf{7}$ and $\mathbf{8}$

\begin{tabular}{cc|c|c|c|c|c|c}
\hline $\mathrm{R}^{1}$ & $\mathrm{R}^{2}$ & $\begin{array}{l}\text { Camphor- } \\
10-\end{array}$ & $\begin{array}{c}\text { Yield } \\
\text { sulfonamide }\end{array}$ & $\begin{array}{c}\text { 2-exo- } \\
\text { alcohol }\end{array}$ & $\begin{array}{c}\text { 2-endo- } \\
\text { alcohol }\end{array}$ & $\begin{array}{c}\text { Yield } \\
\%\end{array}$ & $\begin{array}{c}\text { exo: endo } \\
\%\end{array}$ \\
\hline benzyl & $\mathrm{H}$ & $\mathbf{6 a}$ & 99 & $\mathbf{7 a}$ & $\mathbf{8 a}$ & 95 & $78: 22$ \\
3-chlorophenyl & $\mathrm{H}$ & $\mathbf{6 b}$ & 99 & $\mathbf{7 b}$ & $\mathbf{8 b}$ & 53 & $93: 7$ \\
phenyl & $\mathrm{H}$ & $\mathbf{6 c}$ & 93 & $\mathbf{7 c}$ & $\mathbf{8 c}$ & 97 & $90: 10$ \\
furfuryl & $\mathrm{H}$ & $\mathbf{6 d}$ & 100 & $\mathbf{7 d}$ & $\mathbf{8 d}$ & 83 & $82: 18$ \\
2-pyridyl & $\mathrm{H}$ & $\mathbf{6 e}$ & 84 & $\mathbf{7 e}$ & $\mathbf{8 e}$ & 98 & $87: 13$ \\
1-adamantyl & $\mathrm{H}$ & $\mathbf{6 f}$ & 89 & $\mathbf{7 f}$ & $\mathbf{8 f}$ & 70 & $87: 13$ \\
2-picolyl & $\mathrm{H}$ & $\mathbf{6 g}$ & 90 & $\mathbf{7 g}$ & $\mathbf{8 g}$ & 93 & $90: 10$ \\
$t$-butyl & $\mathrm{H}$ & $\mathbf{6 h}$ & 94 & $\mathbf{7 h}$ & $\mathbf{8 h}$ & 92 & $79: 21$ \\
2-imidazolyl & $\mathrm{H}$ & $\mathbf{6 i}$ & 83 & $\mathbf{7 i}$ & $\mathbf{8 i}$ & 86 & $100: 0$ \\
cyclohexyl & cyclohexyl & $\mathbf{6 j}$ & 88 & $\mathbf{7 j}$ & $\mathbf{8 j}$ & 100 & $62: 38$ \\
\hline
\end{tabular}

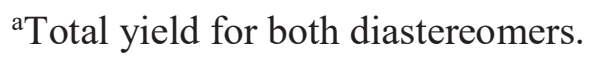

In the present study, attention was focused on using the 2-exo-acryloyloxybornane-10sulfonamides $\mathbf{9 a , b , f}$ as chiral MBH substrates. Pyridinecarbaldehydes tend to react rapidly under $\mathrm{MBH}$ conditions, ${ }^{11}$ and pyridine-4-carbaldehyde and 6-methylpyridine-2-carbaldehyde were selected as the electrophiles and the tertiary amine, DABCO, as the nucleophilic catalyst for these reactions (Scheme 2). The reactions were allowed to run for $90 \mathrm{~h}$ and the desired series of $\mathrm{MBH}$ adducts 12 and 13 were obtained with excellent conversion levels (91-100\%) as determined by ${ }^{1} \mathrm{H}$ NMR analysis after preliminary flash chromatography of the reaction mixtures. The diastereoselectivities were determined by comparing the relative integrals of the signals 
corresponding to the vinylic methylene and hydroxymethine protons (between 5 and $6 \mathrm{ppm}$ ) in the ${ }^{1} \mathrm{H}$ NMR spectra of the mixtures of the major and minor MBH products. The results, summarised in Scheme 2, reveal that while a measure of diastereoselectivity (7-33\% d.e.) was observed in all cases, there is considerable room for improvement, and careful optimisation of the methodology is required to establish reproducibility of our encouraging, preliminary results (up to $95 \%$ d.e.) obtained earlier. ${ }^{6}$ As observed previously, reactions with the more sterically hindered aldehyde, 6methylpyridine-2-carbaldehyde exhibit significantly higher diastereo-selectivities than reactions with pyridine-4-carbaldehyde, confirming the expectation that steric effects play a major role in diastereocontrol. It is expected that future efforts will focus on using inter- and/or intramolecular coordination effects to increase transition-state rigidity and thus enhance diastereoselectivity in such MBH reactions.

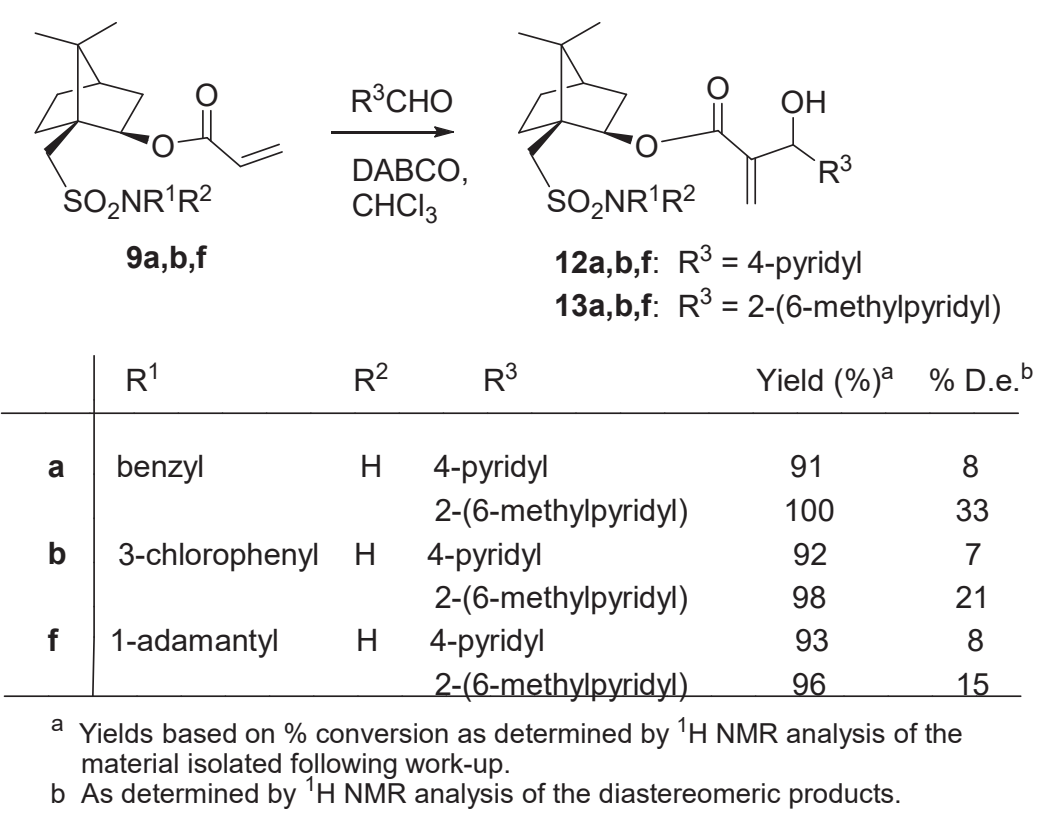

\section{Scheme 2}

\section{Conclusions}

A number of borneol derivatives have been prepared with the potential to serve as chiral auxiliaries in various transformations, and the use of three of these compounds has been explored in asymmetric MBH reactions. Future challenges include the optimisation of reaction conditions and structural modifications to enhance diastereoselectivity. 


\section{Experimental Section}

General. Reagents and solvents were used without further purification. ${ }^{1} \mathrm{H}$ and ${ }^{13} \mathrm{C}$ NMR spectra were recorded on Bruker AMX400 or Avance $\mathrm{II}^{+} 600 \mathrm{MHz}$ spectrometers, and were calibrated using solvent signals; coupling constants are given in Hertz $(\mathrm{Hz})$. Melting points were determined using a hot-stage apparatus, and are uncorrected. IR spectra were recorded on a Perkin Elmer Spectrum 100 FT-IR spectrometer. High-resolution mass spectra were recorded at the University of Stellenbosch Mass Spectrometry Unit. Flash chromatography was carried out using Merck silica gel 60 [230-240 mesh (particle size 0.040-0.063 mm)] and preparative layer chromatography was conducted using silica gel $60 \mathrm{PF}_{254}$.

Compounds $\mathbf{6 a}, \mathbf{b}, \mathbf{c}, \mathbf{e}, \mathbf{f}, \mathbf{g}, \mathbf{h}, \mathbf{j}, \mathbf{7 a}, \mathbf{c}, \mathbf{h}, \mathbf{j}, \mathbf{8 a}, \mathbf{c}, \mathbf{1 2 f}$ and $\mathbf{1 3 f}$ are known. ${ }^{14-17}$ General procedures and analytical data for new compounds are as follows.

\section{Formation of the camphor-10-sulfonamides 6a-j}

General procedure, exemplified by the preparation of $\mathbf{N}$-benzylcamphor-10-sulfonamide (6a). A solution of (+)-camphor-10-sulfonyl chloride (4) (10.0 g, $39.9 \mathrm{mmol})$ in acetonitrile (100 $\mathrm{mL})$ was added dropwise under $\mathrm{N}_{2}$ to a stirred solution of the benzylamine (5a) $(8.67 \mathrm{~mL}, 79.5$ mmol) and DMAP $(1.28 \mathrm{~g}, 10.5 \mathrm{mmol})$ in acetonitrile $(50 \mathrm{~mL})$ at $0{ }^{\circ} \mathrm{C}$. The solution was stirred for $1 \mathrm{~h}$ and water $(50 \mathrm{~mL})$ was then added, followed by $10 \% \mathrm{HCl}(10 \mathrm{~mL})$, and the resulting mixture was extracted with EtOAc $(3 \times 125 \mathrm{~mL})$. The organic layers were combined, washed with $5 \%$ aqueous $\mathrm{NaOH}(25 \mathrm{~mL})$ and dried over anhydrous $\mathrm{MgSO}_{4}$. The solvent was removed in vacuo to afford the known $N$-benzylcamphor-10-sulfonamide (6a) ${ }^{14,15}$ as colourless crystals (12.8 g, 39.8 mmol, 99\%).

$\boldsymbol{N}$-(2-Furfuryl)camphor-10-sulfonamide (6d). Yellow crystals (100\%), mp 42-44 ${ }^{\circ} \mathrm{C}$ (Found: $\mathrm{MNa}^{+}, 334.1085 . \mathrm{C}_{15} \mathrm{H}_{21} \mathrm{NO}_{4} \mathrm{SNa}$ requires, $\left.M+23: 334.1089\right) ; \delta_{\mathrm{H}}\left(600 \mathrm{MHz} ; \mathrm{CDCl}_{3}\right) 0.78(3 \mathrm{H}, \mathrm{s}$, 9-Me), $0.95(3 \mathrm{H}, \mathrm{s}, 8-\mathrm{Me}), 1.42-2.88\left(7 \mathrm{H}\right.$, series of $\left.\mathrm{m}, \mathrm{H}_{2}-3,5,6, \mathrm{H}-4\right), 2.87$ and $3.16(2 \mathrm{H}, \mathrm{AB}$ system, $\left.J 15.0 \mathrm{~Hz}, \mathrm{H}_{2}-10\right), 4.31$ and $4.42\left(2 \mathrm{H}\right.$, two ddd, $J 4.8,7.2$ and $15.6 \mathrm{~Hz}$, furfuryl $\left.\mathrm{CH}_{2}\right), 5.96$ $(1 \mathrm{H}, \mathrm{m}, \mathrm{NH}), 6.32\left(2 \mathrm{H}\right.$, br s, ArH), $7.35(1 \mathrm{H}, \mathrm{s}, \mathrm{ArH}) ; \delta_{\mathrm{C}}\left(150 \mathrm{MHz} ; \mathrm{CDCl}_{3}\right) 19.4(\mathrm{C}-9), 19.9$ (C8), 27.0 and $27.2(\mathrm{C}-3,5), 40.4\left(\mathrm{CH}_{2} \mathrm{~N}\right), 42.8(\mathrm{C}-4), 42.9(\mathrm{C}-6), 48.8(\mathrm{C}-1), 51.3(\mathrm{C}-10), 59.4(\mathrm{C}-$ 7), 108.6, 110.5, 142.6, and 150.4 (ArC), 216.8 (C-2).

$\mathrm{N}$-(2-Imidazolyl)camphor-10-sulfonamide (6h). Brown oily crystals (83\%), m.p. 218-220 ${ }^{\circ} \mathrm{C}$ (Found: $\mathrm{MH}^{+}, 298.1225 . \mathrm{C}_{13} \mathrm{H}_{20} \mathrm{~N}_{3} \mathrm{O}_{3} \mathrm{~S}$ requires, $\left.M+1: 298.1225\right) ; v_{\max }(\mathrm{ATR}) / \mathrm{cm}^{-1} 1675(\mathrm{C}=\mathrm{O})$; $\delta_{\mathrm{H}}\left(400 \mathrm{MHz} ; \mathrm{CDCl}_{3}\right) 0.86(3 \mathrm{H}, \mathrm{s}, 9-\mathrm{Me}), 1.08(3 \mathrm{H}, \mathrm{s}, 8-\mathrm{Me}), 1.44-2.55$ (7H, series of m, $\mathrm{H}_{2}-3,5,6$, H-4), 2.90 and 3.40 (2H, AB system, $\left.J 12.4 \mathrm{~Hz}, \mathrm{H}_{2}-10\right), 6.56$ and $7.23(2 \mathrm{H}$, two s, ArH), $11.6(2 \mathrm{H}$, s, $2 \times \mathrm{NH}) ; \delta_{\mathrm{C}}\left(100 \mathrm{MHz} ; \mathrm{CDCl}_{3}\right) 19.8(\mathrm{C}-8), 19.9(\mathrm{C}-9), 24.7$ and 27.1 (C-5,6), 42.7 (C-4), 43.0 (C-3), 48.2 (C-10), 48.5 (C-1), 59.3 (C-7), 112.6 (imidazolyl C-4',5'), 148.3 (C-2'), 216.5 (C-2).

Formation of $\mathrm{N}$-substituted 2-hydroxybornane-10-sulfonamides 7 and 8. General procedure, exemplified by the preparation of $\mathrm{N}$-benzyl-2-exo-hydroxybornane-10-sulfonamide (7a) and $\mathrm{N}$-benzyl-2-endo-hydroxybornane-10-sulfonamide (8a). A solution of $\mathrm{N}$-benzylcamphor-10sulfonamide (6a) (12.0 g, $37.3 \mathrm{mmol})$ in $\mathrm{EtOH} / \mathrm{H}_{2} \mathrm{O}(5: 1)(104 \mathrm{~mL})$ was added drop-wise to a 
stirred solution of $\mathrm{NaBH}_{4}$ (10 eq.; $14.10 \mathrm{~g}, 372.7 \mathrm{mmol}$ ) in $\mathrm{EtOH} / \mathrm{H}_{2} \mathrm{O} 5: 1(121 \mathrm{~mL})$ at r.t. The mixture was stirred overnight; the reaction was then quenched with $5 \% \mathrm{HCl}(10 \mathrm{~mL})$ and the resulting mixture extracted with EtOAc $(3 \times 25 \mathrm{~mL})$. The organic layers were combined, washed with $5 \%$ brine $(25 \mathrm{~mL})$ and dried over anhydrous $\mathrm{MgSO}_{4}$. Solvent was removed in vacuo to give light-yellow crystalline material (11.5 g), which was chromatographed (HPLC; elution with hexane/EtOAc 8:2) to afford two products.

Fraction 1. The known $N$-benzyl-2-exo-hydroxybornane-10-sulfonamide $7 \mathbf{a}^{14,17}$ as white crystals $(78 \%)$.

Fraction 2. The known $N$-benzyl-2-endo-hydroxybornane-10-sulfonamide $\mathbf{8 a}^{14,17}$ as white crystals $(22 \%)$.

Similar reaction of $N$-(3-chlorophenyl)-10-sulfonamide (6b) afforded two products:

Fraction 1. $\mathbf{N}$-(3-Chlorophenyl)-2-exo-hydroxybornane-10-sulfonamide (7b). White crystals (93\%), mp 108-110 ${ }^{\circ} \mathrm{C}$ [Found: $(\mathrm{M}-\mathrm{H})^{-}$, 342.0916. $\mathrm{C}_{16} \mathrm{H}_{21} \mathrm{ClNO}_{3} \mathrm{~S}$ requires (M-1):: 342.0931]; $v_{\max }(\mathrm{ATR}) / \mathrm{cm}^{-1} 3441(\mathrm{OH}) ; \delta_{\mathrm{H}}\left(600 \mathrm{MHz} ; \mathrm{CDCl}_{3}\right) 0.78(3 \mathrm{H}, \mathrm{s}, 9-\mathrm{Me}), 1.03(3 \mathrm{H}, \mathrm{s}, 8-\mathrm{Me}), 1.14-$ $1.83\left(7 \mathrm{H}\right.$, series of $\left.\mathrm{m}, \mathrm{H}_{2}-3,5,6, \mathrm{H}-4\right), 2.98$ and $3.55\left(2 \mathrm{H}, \mathrm{AB}\right.$ system, $\left.J 13.8 \mathrm{~Hz}, \mathrm{H}_{2}-10\right), 3.05(1 \mathrm{H}$, s, OH), $4.14(1 \mathrm{H}, \mathrm{m}, \mathrm{H}-2), 6.79(1 \mathrm{H}$, br s, NH), $7.12(1 \mathrm{H}$, ddd, $J 0.7,2.3$ and $8.3 \mathrm{~Hz}, \mathrm{ArH}), 7.16$ $(1 \mathrm{H}$, ddd, $J$ 1.1, 2.0 and $8.0 \mathrm{~Hz}, \mathrm{ArH}), 7.23(1 \mathrm{H}, \mathrm{t}, J 2.1 \mathrm{~Hz}, \mathrm{ArH}), 7.29(1 \mathrm{H}, \mathrm{t}, J=8.1 \mathrm{~Hz} \mathrm{~Hz}$, $\mathrm{ArH}) ; \delta_{\mathrm{C}}\left(150 \mathrm{MHz} ; \mathrm{CDCl}_{3}\right) 19.9$ (C-9), 20.5 (C-8), 27.3, 30.5, and 39.3 (C-3,5,6), 44.4 (C-4), 49.0 and 50.5 (C-1,7), 52.0 (C-10), 76.4 (C-2), 118.0, 120.1, 125.3, 130.8, 135.4, and $138.0(\mathrm{ArC})$. Fraction 2. $\mathrm{N}$-(3-Chlorophenyl)-2-endo-hydroxybornane-10-sulfonamide (8b). White crystals (7\%) [Found: $(\mathrm{M}-\mathrm{H})^{-}, 342.0946 . \mathrm{C}_{16} \mathrm{H}_{21} \mathrm{ClNO}_{3} \mathrm{~S}$ requires, $(M-1)^{-}: 342.0931$ ]; $\delta_{\mathrm{H}}(600 \mathrm{MHz}$; $\left.\mathrm{CDCl}_{3}\right) 0.85(6 \mathrm{H}, \mathrm{s}, 8,9-\mathrm{Me}), 1.06-2.44\left(7 \mathrm{H}\right.$, series of $\left.\mathrm{m}, \mathrm{H}_{2}-3,5,6, \mathrm{H}-4\right), 3.13$ and $3.19(2 \mathrm{H}, \mathrm{AB}$ system, $\left.J 14.6 \mathrm{~Hz}, \mathrm{H}_{2}-10\right), 4.32(1 \mathrm{H}, \mathrm{dt}, J 3.9$ and $15.0 \mathrm{~Hz}, \mathrm{H}-2), 7.12-7.28$ (4H, series of m, ArH). Similar reaction of $N$-(furfuryl)camphor-10-sulfonamide (6d) afforded two products:

Fraction 1. $\boldsymbol{N}$-(Furfuryl)-2-exo-hydroxybornane-10-sulfonamide (7d). Yellow crystals (82\%), mp 60-62 ${ }^{\circ} \mathrm{C}$ [Found: (M-H)- 312.1281. $\mathrm{C}_{15} \mathrm{H}_{22} \mathrm{NO}_{4} \mathrm{~S}$ requires, $\left.(M-1):: 312.1270\right] ; v_{\max }(\mathrm{ATR}) / \mathrm{cm}^{-}$ ${ }^{1} 3493(\mathrm{OH}) ; \delta_{\mathrm{H}}\left(400 \mathrm{MHz} ; \mathrm{CDCl}_{3}\right) 0.72(3 \mathrm{H}, \mathrm{s}, 9-\mathrm{Me}), 0.97$ (3H, s, 8-Me), 1.41-1.75 (7H, series of m, $\left.\mathrm{H}_{2}-3,5,6, \mathrm{H}-4\right), 2.97$ and $3.26\left(2 \mathrm{H}, \mathrm{AB}\right.$ system, $\left.J 15.0 \mathrm{~Hz}, \mathrm{H}_{2}-10\right), 3.16$ (1H, br s, OH), 4.04 $(1 \mathrm{H}, \mathrm{m}, \mathrm{H}-2), 4.33\left(2 \mathrm{H}, \mathrm{s}\right.$, furfuryl $\left.\mathrm{CH}_{2}\right), 4.87(1 \mathrm{H}$, br s, $\mathrm{NH}), 6.33(2 \mathrm{H}, \mathrm{m}$, furfuryl H-3',4'), 7.40 (1H, br s, H-5'); $\delta_{\mathrm{C}}\left(100 \mathrm{MHz} ; \mathrm{CDCl}_{3}\right) 19.8$ (C-9), 20.5 (C-8), 27.3 (C-3), 30.3 (C-5), 38.9 (furfuryl $\mathrm{CH}_{2}$ ), 40.0 (C-6), 44.3 (C-4), 48.6 and 50.3 (C-1,7), 53.1 (C-10), 76.4 (C-2), 108.9 and 110.7 (furfuryl C-3',4'), 142.8 (C-5'), 150.0 (C-2').

Fraction 2. $\boldsymbol{N}$-(Furfuryl)-2-endo-hydroxybornane-10-sulfonamide (8d). Brown oil (trace amounts); $\delta_{\mathrm{H}}\left(400 \mathrm{MHz} ; \mathrm{CDCl}_{3}\right) 0.81\left(6 \mathrm{H}\right.$, br s, 8,9-Me), 1.41-1.75 (7H, series of m, $\mathrm{H}_{2}-3,5,6, \mathrm{H}-$ 4), 2.92 and 2.97 (2H, AB system, $\left.J 14.0 \mathrm{~Hz}, \mathrm{H}_{2}-10\right), 3.10$ (1H, br s, OH), 4.16 (1H, m, H-2), 4.34 $\left(2 \mathrm{H}, \mathrm{m}\right.$, furfuryl $\left.\mathrm{CH}_{2}\right), 5.17(1 \mathrm{H}$, br s, $\mathrm{NH}), 6.34$ and $6.36\left(2 \mathrm{H}, \mathrm{m}\right.$, furfuryl $\left.\mathrm{H}-3^{\prime}, 4^{\prime}\right), 7.41(1 \mathrm{H}, \mathrm{m}$, H-5'). 
Similar reaction of $N$-(2-pyridyl)camphor-10-sulfonamide (6e) afforded two products:

Fraction 1. 2-exo-Hydroxy- $\mathrm{N}$-(2-pyridyl)bornane-10-sulfonamide (7e). White crystals (87\%), mp 230-232 ${ }^{\circ} \mathrm{C}$ (Found: $\mathrm{MH}^{+}, 311.1423 . \mathrm{C}_{15} \mathrm{H}_{23} \mathrm{~N}_{2} \mathrm{O}_{3} \mathrm{~S}$ requires, $M+1: 311.1429$ ); $v_{\max }(\mathrm{ATR}) / \mathrm{cm}^{-}$ ${ }^{1} 3413(\mathrm{OH}) ; \delta_{\mathrm{H}}\left(400 \mathrm{MHz} ; \mathrm{CDCl}_{3}\right) 0.81(3 \mathrm{H}, \mathrm{s}, 9-\mathrm{Me}), 1.10(3 \mathrm{H}, \mathrm{s}, 8-\mathrm{Me}), 1.60-1.89$ (7H, series of $\left.\mathrm{m}, \mathrm{H}-4, \mathrm{H}_{2}-3,5,6\right), 3.12$ and $3.74\left(2 \mathrm{H}, \mathrm{AB}\right.$ system, $\left.J 14.1 \mathrm{~Hz}, \mathrm{H}_{2}-10\right), 4.17(1 \mathrm{H}, \mathrm{dd}, J 8.2$ and $4.0 \mathrm{~Hz}, \mathrm{H}-2), 6.84(2 \mathrm{H}$, br s, NH and $\mathrm{OH}), 6.87\left(1 \mathrm{H}, \mathrm{t}, J 6.5 \mathrm{~Hz}\right.$, pyridyl $\left.\mathrm{H}-5{ }^{\prime}\right), 7.65(1 \mathrm{H}, \mathrm{d}, J 8.8$ Hz, H-3'), 7.78 (1H, t, J 7.5 Hz, H-4'), 8.18 (1H, d, J 5.9 Hz, H-6'); $\delta_{\mathrm{C}}\left(150 \mathrm{MHz}\right.$ CDCl $\left.\mathrm{CD}_{3}\right) 20.0$ (C9), 20.5 (C-8), 27.3, 30.5, and 39.4 (C-3,5,6), 44.5 (C-4), 49.1 and 50.7 (C-1,7), 53.5 (C-10), 75.9 (C-2), 115.1 (pyridyl C-3'), 116.3 (C-5'), 140.1 (C-4'), 143.4 (C-6'), 152.1 (C-2').

Fraction 2. 2-endo-Hydroxy- $\mathbf{N}$-(2-pyridyl)bornane-10-sulfonamide (8e). Yellow oil (13\%) (Found: $\mathrm{MH}^{+}, 311.1430 . \mathrm{C}_{15} \mathrm{H}_{23} \mathrm{~N}_{2} \mathrm{O}_{3} \mathrm{~S}$ requires, $\left.M+1: 311.1429\right) ; v_{\max }(\mathrm{ATR}) / \mathrm{cm}^{-1} 3413(\mathrm{OH}) ; \delta_{\mathrm{H}}$ $\left(400 \mathrm{MHz} ; \mathrm{CDCl}_{3}\right) 0.85(3 \mathrm{H}, \mathrm{s}, 9-\mathrm{Me}), 1.15(3 \mathrm{H}, \mathrm{s}, 8-\mathrm{Me}), 1.60-1.85$ (7H, series of m, H-4, $\mathrm{H}_{2}-$ 3,5,6), 2.94 and $3.45\left(2 \mathrm{H}, \mathrm{AB}\right.$ system, $\left.J 13.9 \mathrm{~Hz}, \mathrm{H}_{2}-10\right), 4.40(1 \mathrm{H}, \mathrm{d}, J 9.3 \mathrm{~Hz}, \mathrm{H}-2), 6.84(2 \mathrm{H}, \mathrm{br}$ s, NH and $\mathrm{OH}), 6.74\left(1 \mathrm{H}, \mathrm{t}, J 6.8 \mathrm{~Hz}, \mathrm{H} 5^{\prime}\right), 6.97\left(1 \mathrm{H}, \mathrm{d}, J 8.8 \mathrm{~Hz}, . \mathrm{H}-3^{\prime}\right)$ and 7.69-7.74 (2H, series of m, H-4',6'); $\delta_{\mathrm{C}}\left(150 \mathrm{MHz} ; \mathrm{CDCl}_{3}\right) 20.7$ (C-8,9), 23.6, 28.2, and 38.4 (C-3,5,6), 44.2 (C-4), 48.2 and 51.6 (C-1,7), 54.5 (C-10), 75.1 (C-2), 115.1 (pyridyl C-3'), 116.3 (C-5'), 140.1 (C-4'), 143.4 (C-6'), $152.1\left(\mathrm{C}-2^{\prime}\right)$.

Similar reaction of $\mathrm{N}$-(2-picolyl)camphor-10-sulfonamide $\mathbf{6 g}$ afforded two products.

Fraction 1. 2-exo-Hydroxy- $\mathbf{N}$-(2-picolyl)camphor-10-sulfonamide $\mathbf{7 g}$, as a colourless oil $(90 \%) ; \delta_{\mathrm{H}}\left(400 \mathrm{MHz} ; \mathrm{CDCl}_{3}\right) 0.76(3 \mathrm{H}, \mathrm{s}, 9-\mathrm{Me}), 1.01(3 \mathrm{H}, \mathrm{s}, 8-\mathrm{Me}), 1.08-1.78(7 \mathrm{H}$, series of multiplets, 3-, 5- and 6- $\mathrm{CH}_{2}$ and 4-H), 2.83 and $3.43\left(2 \mathrm{H}, 2 \mathrm{x} \mathrm{d}, J 14.0 \mathrm{~Hz}, 10-\mathrm{CH}_{2}\right)$, and 4.09 $(1 \mathrm{H}, \mathrm{dd}, J 4.0$ and $8.0 \mathrm{~Hz}, 2-\mathrm{H}), 4.46\left(2 \mathrm{H}\right.$, br s, $\left.\mathrm{NCH}_{2}\right), 5.46(1 \mathrm{H}, \mathrm{br} \mathrm{s}, \mathrm{NH}), 7.24(1 \mathrm{H}, \mathrm{m}, \mathrm{ArH})$, $7.31(1 \mathrm{H}, \mathrm{d}, J 8.0 \mathrm{~Hz}, \mathrm{ArH}), 7.71(1 \mathrm{H}, \mathrm{t}, J 7.6 \mathrm{~Hz}, \mathrm{ArH})$ and $8.53(1 \mathrm{H}, \mathrm{d}, J 4.0 \mathrm{~Hz}, \mathrm{ArH}) ; \delta_{\mathrm{C}}(100$ MHz; $\left.\mathrm{CDCl}_{3}\right) 19.9$ (C-8), 20.5 (C-9), 27.4, 30.4 and 39.3 (C-5, C-6 and C-3), 44.4 (C-4), 47.7 (C1), 48.7 (C-7), 50.4 (C-11), 52.5 (C-10), 76.1 (C-2), 122.2, 122.9, 137.2, 149.3 and 155.3 (ArC).

Fraction 2. 2-endo-Hydroxy- $\mathbf{N}$-(2-picolyl)camphor-10-sulfonamide $\mathbf{8 g}$, as a colourless oil (10\%) (Found: $\mathrm{MH}^{+}, 325.1592 . \mathrm{C}_{16} \mathrm{H}_{25} \mathrm{~N}_{2} \mathrm{O}_{3} \mathrm{~S}$ requires, $\left.M+1: 325.1586\right)$; $v_{\max }(\mathrm{ATR}) / \mathrm{cm}^{-1} 3524$ $(\mathrm{OH}) ; \delta_{\mathrm{H}}\left(400 \mathrm{MHz} ; \mathrm{CDCl}_{3}\right) 0.76(3 \mathrm{H}, \mathrm{s}, 9-\mathrm{Me}), 1.01(3 \mathrm{H}, \mathrm{s}, 8-\mathrm{Me}), 1.08-1.78(7 \mathrm{H}$, series of multiplets, 3-, 5- and 6- $\mathrm{CH}_{2}$ and $\left.4-\mathrm{H}\right), 2.83$ and $3.43\left(2 \mathrm{H}, 2 \mathrm{x} \mathrm{d}, J 14.0 \mathrm{~Hz}, 10-\mathrm{CH}_{2}\right)$, and $4.09(1 \mathrm{H}$, dd, $J 4.0$ and $7.8 \mathrm{~Hz}, 2-\mathrm{H}), 4.46\left(2 \mathrm{H}\right.$, br s, $\left.\mathrm{NCH}_{2}\right), 5.46(1 \mathrm{H}$, br s, $\mathrm{NH}), 7.24(1 \mathrm{H}, \mathrm{m}, \mathrm{ArH}), 7.31$ $(1 \mathrm{H}, \mathrm{d}, J 8.0 \mathrm{~Hz}, \mathrm{ArH}), 7.71(1 \mathrm{H}, \mathrm{t}, J 7.6 \mathrm{~Hz}, \mathrm{ArH})$ and $8.53(1 \mathrm{H}, \mathrm{d}, \mathrm{J} 4.0 \mathrm{~Hz}, \mathrm{ArH}) ; \delta_{\mathrm{C}}(100 \mathrm{MHz}$; $\left.\mathrm{CDCl}_{3}\right) 18.9$ (C-8), 20.4 (C-9), 26.7, 30.2 and 38.5 (C-5, C-6 and C-3), 44.0 (C-4), 47.6 (C-1), 47.9 (C-7), 51.1 (C-11), 51.5 (C-10), 75.1 (C-2), 122.9, 122.9, 137.1, 149.2 and 154.9 (ArC).

Similar reaction of $\mathrm{N}$-(2-imidazolyl)camphor-10-sulfonamide (6i) afforded 2-exo-hydroxy- $\mathrm{N}$-(2imidazolyl)bornane-10-sulfonamide (7i) as a black oil (86\%) (Found: $\mathrm{MH}^{+}, 300.1395$. $\mathrm{C}_{13} \mathrm{H}_{22} \mathrm{~N}_{3} \mathrm{O}_{3} \mathrm{~S}$ requires, $\left.M+1: 300.1382\right) ; v_{\max }(\mathrm{ATR}) / \mathrm{cm}^{-1} 3378(\mathrm{OH}) ; \delta_{\mathrm{H}}\left(400 \mathrm{MHz} ; \mathrm{CDCl}_{3}\right) 0.83$ (3H, s, 9-Me), 1.09 (3H, s, 8-Me), 1.22-1.80 (6H, series of m, $\left.\mathrm{H}_{2}-3,5,6\right), 2.17(1 \mathrm{H}, \mathrm{s}, \mathrm{H}-4), 2.87$ and $3.42\left(2 \mathrm{H}, \mathrm{AB}\right.$ system, $\left.J 13.6 \mathrm{~Hz}, \mathrm{H}_{2}-10\right), 3.25(1 \mathrm{H}, \mathrm{s}, \mathrm{OH}), 3.93(1 \mathrm{H}, \mathrm{m}, \mathrm{H}-2), 6.53(2 \mathrm{H}, \mathrm{br}$ s, imidazolyl H-4',5'), 11.60 (2H, s, NH); $\delta_{\mathrm{C}}\left(100 \mathrm{MHz} ; \mathrm{CDCl}_{3}\right) 19.8$ (C-8), 20.4 (C-9), 27.1, 30.7, 
and $30.9(\mathrm{C}-3,5,6), 44.1(\mathrm{C}-4), 47.3(\mathrm{C}-7), 50.1(\mathrm{C}-1), 50.6(\mathrm{C}-10), 75.8(\mathrm{C}-2), 106.7$ and 113.5 (imidazolyl C-4',5'), 147.6 (C-2').

Similar reaction of $N, N$-dicyclohexylcamphor-10-sulfonamide (6j) afforded two products:

Fraction 1. The known $N, N$-dicyclohexyl-2-exo-hydroxybornane-10-sulfonamide $(7 \mathbf{j})^{7}$ as white crystals $(62 \%)$.

Fraction 2. $\mathbf{N}, \mathbf{N}$-Dicyclohexyl-2-endo-hydroxybornane-10-sulfonamide (8j). White crystals (38\%), mp 162-164 ${ }^{\circ} \mathrm{C}$ (Found: $\mathrm{MH}^{+}$, 398.2725. $\mathrm{C}_{22} \mathrm{H}_{40} \mathrm{NO}_{3} \mathrm{~S}$ requires, $M+1$ : 398.2729); $v_{\max }$ $(\mathrm{ATR}) / \mathrm{cm}^{-1} 3503(\mathrm{OH}) ; \delta_{\mathrm{H}}\left(400 \mathrm{MHz} ; \mathrm{CDCl}_{3}\right) 0.87$ and $0.88(6 \mathrm{H}$, two s, 8,9-Me), 1.11-3.27 $(31 \mathrm{H}$, m, $\mathrm{H}_{2}-3,5,6,10,2$ x cyclohexyl), $3.82(1 \mathrm{H}, \mathrm{m}, \mathrm{OH}), 4.29(1 \mathrm{H}, \mathrm{m}, \mathrm{H}-2) ; \delta_{\mathrm{C}}\left(100 \mathrm{MHz} ; \mathrm{CDCl}_{3}\right) 20.0$ and $20.6(\mathrm{C}-8,9), 44.0(\mathrm{C}-4), 57.8(2 \times \mathrm{CHN}), 75.5(\mathrm{C}-2), 25.1,26.4,32.7$, and 32.8 (cyclohexyl $\left.\mathrm{CH}_{2}\right), 27.4,33.5,38.1$, and $59.2(\mathrm{C}-3,5,6,10), 49.7$ and $51.6(\mathrm{C}-1,7)$.

Formation of the $\mathrm{N}$-substituted 2-(acryloyloxy)bornane-10-sulfonamides 9-11. General procedure, exemplified by the preparation of 2-exo-acryloyloxy- $N$-benzylbornane-10sulfonamide (9a) and 2-endo-acryloyloxy- $N$-benzylbornane-10-sulfonamide (10a). Neutral $\mathrm{Al}_{2} \mathrm{O}_{3}(0.244 \mathrm{~g}, 2.4 \mathrm{mmol})$ was added to the mixture of the diastereomeric alcohols $7 \mathbf{a}$ and $8 \mathbf{a}(0.50$ $\mathrm{g}, 1.5 \mathrm{mmol})$, followed by acryloyl chloride $(0.30 \mathrm{~g}, 3.3 \mathrm{mmol})$. The resulting dispersion was shaken, sealed and kept unstirred at r.t. for $72 \mathrm{~h}$. The mixture was then taken up in $\mathrm{CHCl}_{3}(3 \times 1$ $\mathrm{mL}$ ), filtered and the filtrate dried over anhydrous $\mathrm{MgSO}_{4}$. The solvent was removed in vacuo to give an oil which was then treated with triethylamine $(0.46 \mathrm{~g}, 4.5 \mathrm{mmol})$. The resulting mixture was stored under $\mathrm{N}_{2}$ and stirred at $25^{\circ} \mathrm{C}$ for $1 \mathrm{~h}$ and then taken up in EtOAc $(5 \mathrm{~mL})$; the organic solution was washed with brine and dried over anhydrous $\mathrm{MgSO}_{4}$. Solvent was removed in vacuo to give an oil which was chromatographed [HPLC; elution with hexane-EtOAc (8: 2)] to afford three products.

Fraction 1. 2-exo-Acryloyloxy- $\mathrm{N}$-benzylbornane-10-sulfonamide (9a). White crystals $(0.39 \mathrm{~g}$, 1.0 mmol, 67\%), mp 82-84 ${ }^{\circ} \mathrm{C}$ (Found: $\mathrm{MH}^{+}, 378.1747 . \mathrm{C}_{20} \mathrm{H}_{28} \mathrm{NO}_{4} \mathrm{~S}$ requires, $M+1: 378.1747$ ); $v_{\max }(\mathrm{ATR}) / \mathrm{cm}^{-1} 1706(\mathrm{C}=\mathrm{O}) ; \delta_{\mathrm{H}}\left(400 \mathrm{MHz} ; \mathrm{CDCl}_{3}\right) 0.82$ and $0.99(6 \mathrm{H}$, two s, 8,9-Me), 1.18-2.03 (7H, series of m, $\left.\mathrm{H}_{2}-3,5,6, \mathrm{H}-4\right), 2.81$ and $3.43\left(2 \mathrm{H}, \mathrm{AB}\right.$ system, $\left.J 14.0 \mathrm{~Hz}, \mathrm{H}_{2}-10\right), 4.25(2 \mathrm{H}, \mathrm{d}, J$ $\left.6.0 \mathrm{~Hz}, \mathrm{PhCH}_{2}\right), 4.57\left(1 \mathrm{H}\right.$, br s, NH), $5.04(1 \mathrm{H}, \mathrm{m}, \mathrm{H}-2), 5.79\left(1 \mathrm{H}, \mathrm{d}, J_{\text {cis }} 10.4 \mathrm{~Hz}, \mathrm{H}_{\mathrm{E}}-3^{\prime}\right), 6.09$ $\left(1 \mathrm{H}, \mathrm{dd}, J 10.2\right.$ and $\left.17.4 \mathrm{~Hz}, \mathrm{H}-2^{\prime}\right), 6.33\left(1 \mathrm{H}, \mathrm{d}, J_{\text {trans }} 17.6 \mathrm{~Hz}, \mathrm{~Hz}_{\mathrm{Z}} 3^{\prime}\right), 7.33(5 \mathrm{H}$, overlapping signals, $\mathrm{ArH}) ; \delta_{\mathrm{C}}\left(100 \mathrm{MHz} ; \mathrm{CDCl}_{3}\right) 19.9$ and 20.3 (C-8,9), 27.0, 29.8, and 39.5 (C-3,5,6), 44.4 (C-4), 47.2 $\left(\mathrm{PhCH}_{2}\right), 49.2$ and 49.4 (C-1,7), 51.9 (C-10), 77.9 (C-2), 128.9 (C-2'), 130.3 (C-3'), 127.97, 128.0, 128.8, and 137.0 (ArC), $164.8\left(\mathrm{C}-1^{\prime}\right)$.

Fraction 2. $\mathbf{N}$-Benzyl-2-exo-[(3-chloropropanoyl)oxy]bornane-10-sulfonamide (11a). White crystals (0.13 g, $0.3 \mathrm{mmol}, 20 \%$ ), mp $92-94{ }^{\circ} \mathrm{C}$ (Found: $\mathrm{MH}^{+}$, 414.1498. $\mathrm{C}_{20} \mathrm{H}_{29} \mathrm{ClNO}_{4} \mathrm{~S}$ requires, $M+1: 414.1506) ; v_{\max }(\mathrm{ATR}) / \mathrm{cm}^{-1} 1728(\mathrm{C}=\mathrm{O}) ; \delta_{\mathrm{H}}\left(400 \mathrm{MHz} ; \mathrm{CDCl}_{3}\right) 0.79$ and $0.96(6 \mathrm{H}$, two s, 8,9-Me), 1.18-1.96 (7H, series of $\left.\mathrm{m}, \mathrm{H}_{2}-3,5,6, \mathrm{H}-4\right), 2.74\left(2 \mathrm{H}, \mathrm{m}, \mathrm{H}_{2}-2^{\prime}\right), 2.76$ and $3.55(2 \mathrm{H}, \mathrm{AB}$ system, $\left.J 14.0 \mathrm{~Hz}, \mathrm{H}_{2}-10\right), 3.71\left(2 \mathrm{H}, \mathrm{m}, \mathrm{H}_{2}-3\right.$ '), 4.29 (2H, d, J $\left.6.0 \mathrm{~Hz}, \mathrm{PhCH}_{2}\right), 4.66(1 \mathrm{H}, \mathrm{s}, \mathrm{NH})$, $4.98(1 \mathrm{H}, \mathrm{m}, \mathrm{H}-2), 7.36(5 \mathrm{H}$, overlapping signals, $\mathrm{ArH}) ; \delta_{\mathrm{C}}\left(100 \mathrm{MHz} ; \mathrm{CDCl}_{3}\right) 19.9$ and $20.2(\mathrm{C}-$ 8,9), 27.0, 30.0, and $39.4(\mathrm{C}-3,5,6), 37.9\left(\mathrm{C}-2^{\prime}\right), 39.2\left(\mathrm{C}-3^{\prime}\right), 44.4(\mathrm{C}-4), 47.2\left(\mathrm{PhCH}_{2}\right), 49.1$ and 49.4 (C-1,7), 52.2 (C-10), 78.5 (C-2), 128.0, 128.1, 128.9, and 137.0 (ArC), 169.0 (C-1'). 
Fraction 3. 2-endo-Acryloyloxy- $N$-benzylbornane-10-sulfonamide (10a) as a solid isolated in trace amounts as a mixture with compound $11 \mathrm{a} ; \delta_{\mathrm{H}}\left(400 \mathrm{MHz} ; \mathrm{CDCl}_{3}\right) 0.85$ and $0.86(6 \mathrm{H}$, two s, 8,9-Me), 1.19-1.98 (7H, series of m, H2-3,5,6, H-4), 2.92 (2H, AB system, $J 14.4$ Hz, H2-10), 4.27 $\left(2 \mathrm{H}, \mathrm{d}, J 6.0 \mathrm{~Hz}, \mathrm{PhC}_{2}\right), 5.16(1 \mathrm{H}, \mathrm{d}, J 6.0 \mathrm{~Hz}, \mathrm{NH}), 5.16\left(1 \mathrm{H}, \mathrm{d}, J_{\text {cis }} 10.4 \mathrm{~Hz}, \mathrm{H}_{\mathrm{E}}-3^{\prime}\right), 5.79(1 \mathrm{H}$, m, H-2), $6.10\left(1 \mathrm{H}, \mathrm{dd}, J 10.4\right.$ and $\left.17.2 \mathrm{~Hz}, \mathrm{H}-2{ }^{\prime}\right), 6.42\left(1 \mathrm{H}, \mathrm{d}, J_{\text {trans }} 17.2 \mathrm{~Hz}, \mathrm{H}_{\mathrm{Z}}-3^{\prime}\right), 7.31-7.37(5 \mathrm{H}$, overlapping signals, ArH).

Similar reaction of crude $N$-(3-chlorophenyl)-2-exo-hydroxybornane-10-sulfonamide (7b) afforded two products:

Fraction 1. 2-exo-Acryloyloxy- $\mathbf{N}$-(3-chlorophenyl)bornane-10-sulfonamide (9b) as white crystals $(0.420 \mathrm{~g}, 1.1 \mathrm{mmol}, 73 \%), \mathrm{mp} 150-152{ }^{\circ} \mathrm{C}$ (Found: $\mathrm{MH}^{+}, 398.1195 . \mathrm{C}_{19} \mathrm{H}_{25} \mathrm{ClNO}_{4} \mathrm{~S}$ requires, $M+1$ : 398.1193$)$; $v_{\max }(\mathrm{ATR}) / \mathrm{cm}^{-1} 1710(\mathrm{C}=\mathrm{O}) ; \delta_{\mathrm{H}}\left(400 \mathrm{MHz} ; \mathrm{CDCl}_{3}\right) 0.86$ and $1.00(6 \mathrm{H}$, two s, 8,9-Me), 1.26-2.02 (7H, series of $\left.\mathrm{m}, \mathrm{H}_{2}-3,5,6, \mathrm{H}-4\right), 3.03$ and $3.62(2 \mathrm{H}, \mathrm{AB}$ system, $J 14.0$ $\left.\mathrm{Hz}, \mathrm{H}_{2}-10\right), 5.07(1 \mathrm{H}, \mathrm{m}, \mathrm{H}-2), 5.75\left(1 \mathrm{H}, \mathrm{d}, J_{\text {cis }} 10.4 \mathrm{~Hz}, \mathrm{H}_{\mathrm{E}}-3^{\prime}\right), 5.98(1 \mathrm{H}, \mathrm{dd}, J 10.4$ and $17.2 \mathrm{~Hz}$, H-2'), $6.24\left(1 \mathrm{H}, \mathrm{d}, J_{\text {trans }} 17.2 \mathrm{~Hz}, \mathrm{~Hz}-3^{\prime}\right), 7.05-7.21$ (5H, overlapping signals, ArH and NH); $\delta_{\mathrm{C}}$ $\left(100 \mathrm{MHz} ; \mathrm{CDCl}_{3}\right) 19.9$ and 20.3 (C-8,9), 27.1, 30.1, and 39.5 (C-3,5,6), 44.4 (C-4), 49.2 and 49.7 (C-1,7), 50.7 (C-10), 77.7 (C-2), 117.0, 119.1, 124.5, 130.6, 135.3, and 138.5 (ArC), 128.5 and $130.4\left(\mathrm{C}-2^{\prime}, 3^{\prime}\right), 164.8\left(\mathrm{C}-1^{\prime}\right)$.

Fraction 2. $\mathrm{N}$-(3-Chlorophenyl)-2-exo-[(3-chloropropanoyl)oxy]bornane-10-sulfonamide (11b), white crystals $(0.100 \mathrm{~g}, 0.2 \mathrm{mmol}, 13 \%), \mathrm{mp} 120-122^{\circ} \mathrm{C} ; v_{\max }(\mathrm{ATR}) / \mathrm{cm}^{-1} 1698(\mathrm{C}=\mathrm{O}) ; \delta_{\mathrm{H}}$ $\left(400 \mathrm{MHz} ; \mathrm{CDCl}_{3}\right) 0.84$ and $0.99\left(6 \mathrm{H}\right.$, two s, 8,9-Me), 1.67-1.99 (7H, series of m, $\left.\mathrm{H}_{2}-3,5,6, \mathrm{H}-4\right)$, $2.68\left(2 \mathrm{H}, \mathrm{t}, J 6.6 \mathrm{~Hz}, \mathrm{H}_{2}-2^{\prime}\right), 3.01$ and $3.55\left(2 \mathrm{H}, \mathrm{AB}\right.$ system, $\left.J 14.0 \mathrm{~Hz}, \mathrm{H}_{2}-10\right), 3.66\left(2 \mathrm{H}, \mathrm{m}, \mathrm{H}_{2}-\right.$ 3'), $5.02(1 \mathrm{H}, \mathrm{m}, \mathrm{H}-2), 6.69(1 \mathrm{H}$, br s, NH), $7.06(1 \mathrm{H}, \mathrm{ddd}, J 0.9,2.1$ and $8.1 \mathrm{~Hz}, \mathrm{ArH}), 7.14(1 \mathrm{H}$, ddd, $J 0.8,2.0$ and $8.0 \mathrm{~Hz}, \mathrm{ArH}), 7.23(1 \mathrm{H}, \mathrm{t}, J 2.0 \mathrm{~Hz}, \mathrm{ArH}), 7.28(1 \mathrm{H}, \mathrm{t}, J 8.0 \mathrm{~Hz}, \mathrm{ArH}) ; \delta_{\mathrm{C}}(100$ $\left.\mathrm{MHz} ; \mathrm{CDCl}_{3}\right) 19.9$ and 20.3 (C-8,9), 27.0, 30.2, and 39.5 (C-3,5,6), 37.7 (C-2'), 39.1 (C-3'), 44.4 (C-4), 49.1 and 49.7 (C-1,7), 50.9 (C-10), 78.4 (C-2), 117.7, 119.6, 125.0, 130.7, 135.4, and 138.3 (ArC), 168.9 (C-1').

Similar reaction of crude 2-exo-hydroxy- $N$-phenylbornane-10-sulfonamide (7c) afforded two fractions:

Fraction 1. 2-exo-Acryloyloxy- $\boldsymbol{N}$-phenylbornane-10-sulfonamide (9c) (36\%, contaminated with compound 11c); $\delta_{\mathrm{H}}\left(400 \mathrm{MHz} ; \mathrm{CDCl}_{3}\right) 0.82$ and $0.96(6 \mathrm{H}$, two s, 8,9-Me), 1.20-2.03 (7H, series of $\left.\mathrm{m}, \mathrm{H}_{2}-3,5,6, \mathrm{H}-4\right), 3.01$ and $3.54\left(2 \mathrm{H}, \mathrm{AB}\right.$ system, $\left.J 14.0 \mathrm{~Hz}, \mathrm{H}_{2}-10\right), 5.01(1 \mathrm{H}, \mathrm{d}, J 6.4$ Hz, H-2), $5.73\left(1 \mathrm{H}, \mathrm{d}, J_{\text {cis }} 12.0 \mathrm{~Hz}, \mathrm{H}_{\mathrm{E}}-3^{\prime}\right), 5.97\left(1 \mathrm{H}, \mathrm{dd}, J 10.4\right.$ and $\left.17.2 \mathrm{~Hz}, \mathrm{H}-2^{\prime}\right), 6.22(1 \mathrm{H}, \mathrm{d}$, $\left.J_{\text {trans }} 17.2 \mathrm{~Hz}, \mathrm{~Hz}_{\mathrm{z}} 3^{\prime}\right), 7.10-7.34(5 \mathrm{H}, \mathrm{m}, \mathrm{ArH}) ; \delta_{\mathrm{C}}\left(100 \mathrm{MHz} ; \mathrm{CDCl}_{3}\right) 19.8$ and $20.2(\mathrm{C}-8,9), 27.0$, 30.0, and $39.4(\mathrm{C}-3,5,6), 44.3(\mathrm{C}-4), 49.0$ and 49.6 (C-1,7), 50.1 (C-10), $78.3(\mathrm{C}-2), 128.6$ and 130.3 (C-2',3'), 119.1, 119.2, 120.1, 124.9, 129.6, and 137.1 (ArC), 169.0 (C-1').

Fraction 2. 2-exo-[(3-Chloropropanoyl)oxy]- $N$-phenylbornane-10-sulfonamide (11c) (64\%, contaminated with compound $9 \mathrm{c}) ; \delta_{\mathrm{H}}\left(400 \mathrm{MHz} ; \mathrm{CDCl}_{3}\right) 0.83$ and $1.00(6 \mathrm{H}$, two s, 8,9-Me), 1.20 $2.03\left(7 \mathrm{H}\right.$, series of m, $\left.\mathrm{H}_{2}-3,5,6, \mathrm{H}-4\right), 2.58\left(1 \mathrm{H}, \mathrm{t}, J 6.8 \mathrm{~Hz}, \mathrm{CO} . \mathrm{H}_{\mathrm{a}}\right), 3.01\left(1 \mathrm{H}, \mathrm{m}, J 6.8 \mathrm{~Hz}, \mathrm{CO} . \mathrm{H}_{\mathrm{b}}\right)$, 3.03 and $3.54\left(2 \mathrm{H}, \mathrm{AB}\right.$ system, $\left.J 14.0 \mathrm{~Hz}, \mathrm{H}_{2}-10\right), 3.61\left(2 \mathrm{H}, \mathrm{d}, J 7.0 \mathrm{~Hz}, \mathrm{H}_{2}-3^{\prime}\right), 5.08(1 \mathrm{H}, \mathrm{d}, J 6.4$ $\mathrm{Hz}, \mathrm{H}-2), 7.10-7.34$ (5H, m, ArH); $\delta_{\mathrm{C}}\left(100 \mathrm{MHz} ; \mathrm{CDCl}_{3}\right) 19.9$ and 20.2 (C-8,9), 27.0, 29.9, and 
39.5 (C-3,5,6), 37.6 and $39.0\left(\mathrm{C}^{2} 2^{\prime}, 3^{\prime}\right), 44.4(\mathrm{C}-4), 49.1$ and $49.6(\mathrm{C}-1,7), 50.1$ (C-10), 77.8 (C-2), $119.2(2 \times \mathrm{ArC}), 120.1$ (ArC), $129.6(2 \times \mathrm{ArC}), 137.2(\mathrm{ArC}), 164.8\left(\mathrm{C}-1^{\prime}\right)$.

Similar reaction of crude 2-exo-hydroxy- $N$-(2-pyridinyl)bornane-10-sulfonamide (7e) afforded two products:

Fraction 1. 2-exo-Acryloyloxy- $\mathrm{N}$-(2-pyridinyl)bornane-10-sulfonamide (9e) (37\%), yellow solid contaminated with compound 11e; $\delta_{\mathrm{H}}\left(400 \mathrm{MHz} ; \mathrm{CDCl}_{3}\right) 0.82(3 \mathrm{H}, \mathrm{s}, 9-\mathrm{Me}), 0.85(3 \mathrm{H}, \mathrm{s}, 8-$ Me), 1.24-2.17 (7H, series of $\left.\mathrm{m}, \mathrm{H}_{2}-3,5,6, \mathrm{H}-4\right), 3.06$ and 3.57 (2H, AB system, $J 14.4 \mathrm{~Hz}, \mathrm{H}_{2}-10$ ), $5.07(1 \mathrm{H}, \mathrm{m}, \mathrm{H}-2), 5.73\left(1 \mathrm{H}, \mathrm{d}, J_{\text {cis }} 10.4 \mathrm{~Hz}, \mathrm{H}_{\mathrm{E}}-3^{\prime}\right), 5.98\left(1 \mathrm{H}, \mathrm{dd}, J 10.4\right.$ and $\left.17.6 \mathrm{~Hz}, \mathrm{H}-2^{\prime}\right), 6.26$ $\left(1 \mathrm{H}, \mathrm{d}, J_{\text {trans }} 17.6 \mathrm{~Hz}, \mathrm{H}_{\mathrm{Z}}-3^{\prime}\right), 6.55(1 \mathrm{H}, \mathrm{br} \mathrm{s}, \mathrm{NH}), 7.43-7.75$ (4H, series of m, ArH).

Fraction 2. 2-exo-(3-Chloropropanoyloxy)- $\mathrm{N}$-(2-pyridinyl)bornane-10-sulfonamide (11e) $(63 \%)$, yellow solid contaminated with compound $9 \mathrm{e} ; \delta_{\mathrm{H}}\left(400 \mathrm{MHz} ; \mathrm{CDCl}_{3}\right) 0.91(3 \mathrm{H}, \mathrm{s}, 9-\mathrm{Me})$, $1.01(3 \mathrm{H}, \mathrm{s}, 8-\mathrm{Me}), 1.24-2.17$ (7H, series of $\left.\mathrm{m}, \mathrm{H}_{2}-3,5,6, \mathrm{H}-4\right), 3.04$ and 3.57 (2H, AB system, $J$ 14.4 Hz, H2-10), 2.93 (2H, m, H2-2'), 4.33 (2H, m, H2-3'), 5.07 (1H, m, H-2), 6.48 (1H, br s, NH), 7.43-7.75 (4H, series of $\mathrm{m}, \mathrm{ArH})$.

Similar reaction of crude $\mathrm{N}$-(1-adamantyl)-2-exo-hydroxybornane-10-sulfonamide $\mathbf{7 f}$ afforded three products:

Fraction 1. The known 2-exo-acryloyloxy- $N$-(1-adamantyl)bornane-10-sulfonamide $(\mathbf{9 f})^{6}$ as white crystals $(60 \%)$.

Fraction 2. 2-endo-Acryloyloxy- $\boldsymbol{N}$-(1-adamantyl)bornane-10-sulfonamide (10f) (contaminated with compound 11f); $\delta_{\mathrm{H}}\left(400 \mathrm{MHz} ; \mathrm{CDCl}_{3}\right) 0.90$ and $1.03(6 \mathrm{H}$, two s, 8,9-Me), 1.18-2.01 $\left(6 \mathrm{H}\right.$, series of $\left.\mathrm{m}, \mathrm{H}_{2}-3,5,6\right), 1.60$ and $1.92\left(12 \mathrm{H}\right.$, two $\mathrm{m}$, adamantyl $\left.\mathrm{CH}_{2}\right), 1.79(1 \mathrm{H}, \mathrm{m}$, $\mathrm{H}-4), 2.07$ (3H, m, adamantyl CH), 2.91 and $3.56\left(2 \mathrm{H}, \mathrm{AB}\right.$ system, $\left.J 14.0 \mathrm{~Hz}, \mathrm{H}_{2}-10\right), 4.06(1 \mathrm{H}, \mathrm{s}$, $\mathrm{NH}), 5.06(1 \mathrm{H}, \mathrm{m}, \mathrm{H}-2), 5.79\left(1 \mathrm{H}, \mathrm{d}, J_{\text {cis }} 10.4 \mathrm{~Hz}, \mathrm{H}_{\mathrm{E}}-3^{\prime}\right), 6.10\left(1 \mathrm{H}, \mathrm{dd}, J 10.4\right.$ and $\left.17.2 \mathrm{~Hz}, \mathrm{H}-2^{\prime}\right)$, $6.35\left(1 \mathrm{H}, \mathrm{d}, J_{\text {trans }} 17.2 \mathrm{~Hz}, \mathrm{~Hz}_{-}{ }^{\prime}\right) ; \delta_{\mathrm{C}}\left(100 \mathrm{MHz} ; \mathrm{CDCl}_{3}\right) 20.0$ and $20.4(\mathrm{C}-8,9), 27.1,29.9$, and 39.5 (C-3,5,6), 29.6 (adamantyl $\mathrm{CH}), 35.9$ and 43.4 (adamantyl $\left.\mathrm{CH}_{2}\right), 44.4(\mathrm{C}-4), 49.3$ and 49.5 (C-1,7), 55.1 (C-10), 78.1 (C-2), 129.0 (C-2'), 130.0 (C-3'), 164.7 (C-1').

Fraction 3. 2-exo-[(3-Chloropropanoyl)oxy]- $\mathrm{N}$-(adamantyl)bornane-10-sulfonamide (11f). White crystals $(0.33 \mathrm{~g}, 34 \%), \mathrm{mp} 146-148{ }^{\circ} \mathrm{C} ; \delta_{\mathrm{H}}\left(400 \mathrm{MHz} ; \mathrm{CDCl}_{3}\right) 0.90$ and $1.03(6 \mathrm{H}, 2 \times \mathrm{s}, 8-$ and 9-Me), 1.26 - 2.02 (6H, series of $\left.\mathrm{m}, \mathrm{H}_{2}-3,5,6\right), 1.67$ and 1.95 (12H, two m, 12-, 14-, 16-, 17-, 19- and 20- $\left.\mathrm{CH}_{2}\right), 1.79(1 \mathrm{H}, \mathrm{m}, 4-\mathrm{H}), 2.11(3 \mathrm{H}, \mathrm{m}, 13-, 15-$ and $18-\mathrm{CH}), 2.78(2 \mathrm{H}, \mathrm{t}, J 6.4$ and 6.8 $\left.\mathrm{Hz}, 2^{\prime}-\mathrm{CH}_{2}\right), 3.21\left(2 \mathrm{H}, 2 \times \mathrm{d}, J 14.0 \mathrm{~Hz}, 10-\mathrm{CH}_{2}\right), 3.75\left(2 \mathrm{H}, \mathrm{m}, 3^{\prime}-\mathrm{CH}_{2}\right), 4.02(1 \mathrm{H}, \mathrm{s}, \mathrm{NH})$ and 5.00 $(1 \mathrm{H}, \mathrm{d}, J 5.6 \mathrm{~Hz}, 2-\mathrm{CH}) ; \delta_{\mathrm{C}}\left(100 \mathrm{MHz} ; \mathrm{CDCl}_{3}\right) 20.0$ and 20.4 (C-8 and C-9), 27.1, 30.1 and 39.5 (C-3, C-5 and C-6), 29.6 (C-13, C-15 and C-18), 36.0 (C-14, C-19 and C-20), 43.4 (C-12, C-16 and C-17), 38.0 (C-2'), $39.2\left(\mathrm{C}-3^{\prime}\right), 44.5$ (C-4), 49.4 and 49.5 (C-1 and C-7), 55.1 (C-11), 55.6 (C$10), 78.1(\mathrm{C}-2)$ and $168.8(\mathrm{C}=\mathrm{O})$.

Similar reaction of crude $N$-t-butyl-2-exo-hydroxybornane-10-sulfonamide $\mathbf{7 h}$ afforded two fractions:

Fraction 1. 2-exo-Acryloyloxy- $\mathbf{N}$-t-butylbornane-10-sulfonamide 9 h. Colourless oil (0.86 g, 86\%) (Found: $\mathrm{MH}^{+}, 344.1897 . \mathrm{C}_{17} \mathrm{H}_{30} \mathrm{NO}_{4} \mathrm{~S}$ requires, $\left.\mathrm{M}+\mathrm{H}: 344.1896\right) ; \delta_{\mathrm{H}}\left(400 \mathrm{MHz} ; \mathrm{CDCl}_{3}\right)$ 0.90 and $1.03(6 \mathrm{H}, 2 \times \mathrm{s}, 8-$ and $9-\mathrm{Me}), 1.34\left[9 \mathrm{H}, \mathrm{s}, \mathrm{C}\left(\mathrm{CH}_{3}\right)_{3}\right], 1.21-1.99\left(7 \mathrm{H}\right.$, series of $\mathrm{m}, \mathrm{H}_{2}-$ 
3,5,6, H-4), 2.91 and $3.54\left(2 \mathrm{H}, 2 \times \mathrm{d}, J 14.0 \mathrm{~Hz}, 10-\mathrm{CH}_{2}\right), 4.12(1 \mathrm{H}, \mathrm{s}, \mathrm{NH}), 5.05(1 \mathrm{H}, \mathrm{d}, J 7.2 \mathrm{~Hz}$, 2-H), $5.79\left(1 \mathrm{H}, \mathrm{d}, J 10.4 \mathrm{~Hz}, 3^{\prime}-\mathrm{H}_{\mathrm{E}}\right), 6.09\left(1 \mathrm{H}, \mathrm{dd}, J 10.4\right.$ and $\left.17.2 \mathrm{~Hz}, 2^{\prime}-\mathrm{H}\right)$ and $6.34(1 \mathrm{H}, \mathrm{d}, J$ $\left.17.2 \mathrm{~Hz}, 3^{\prime}-\mathrm{Hz}\right) ; \delta_{\mathrm{C}}\left(100 \mathrm{MHz} ; \mathrm{CDCl}_{3}\right) 20.0$ and 20.4 (C-8 and C-9), 27.1, 29.9 and 39.5 (C-3, C5 and $\mathrm{C}-6), 30.3\left[\mathrm{C}\left(\mathrm{CH}_{3}\right)\right], 44.4(\mathrm{C}-4), 49.4$ and $54.7(\mathrm{C}-7$ and $\mathrm{C}-1), 54.8\left[\mathrm{C}_{\left.\left(\mathrm{CH}_{3}\right)_{3}\right]} 58.9(\mathrm{C}-10)\right.$, $78.0(\mathrm{C}-2), 129.0\left(\mathrm{C}-2^{\prime}\right), 130.1\left(\mathrm{C}-3^{\prime}\right)$ and $164.7(\mathrm{C}=\mathrm{O})$.

Fraction 2. 2-endo-Acryloyloxy- $\mathrm{N}$-t-butylbornane-10-sulfonamide $10 \mathrm{~h}$. Colourless oil $(0.14 \mathrm{~g}$, 14\%) (Found: $\mathrm{MH}^{+}, 344.1892 . \mathrm{C}_{17} \mathrm{H}_{30} \mathrm{NO}_{4} \mathrm{~S}$ requires, $\left.M+\mathrm{H}: 344.1896\right) ; \delta_{\mathrm{H}}\left(400 \mathrm{MHz} ; \mathrm{CDCl}_{3}\right)$ 0.94 and $0.98\left(6 \mathrm{H}, 2 \times \mathrm{s}, 8\right.$ - and 9-Me), $1.33\left[9 \mathrm{H}, \mathrm{s}, \mathrm{C}\left(\mathrm{CH}_{3}\right)_{3}\right], 1.07-1.78\left(7 \mathrm{H}\right.$, series of $\mathrm{m}, \mathrm{H}_{2}-$ $3,5,6, \mathrm{H}-4), 3.06$ and $3.16\left(2 \mathrm{H}, 2 \times \mathrm{d}, J 14.0 \mathrm{~Hz}, 10-\mathrm{CH}_{2}\right), 4.01(1 \mathrm{H}, \mathrm{s}, \mathrm{NH}), 5.05(1 \mathrm{H}, \mathrm{d}, J 9.6 \mathrm{~Hz}$, 2-H), $5.86\left(1 \mathrm{H}, \mathrm{d}, J 10.8 \mathrm{~Hz}, 3^{\prime}-\mathrm{H}_{\mathrm{E}}\right), 6.15\left(1 \mathrm{H}, \mathrm{dd}, J 10.8\right.$ and $\left.17.2 \mathrm{~Hz}, 2^{\prime}-\mathrm{H}\right)$ and $6.47(1 \mathrm{H}, \mathrm{d}, J$ $17.2 \mathrm{~Hz}, 3^{\prime}-\mathrm{Hz}_{\mathrm{z}}$; $\delta_{\mathrm{C}}\left(100 \mathrm{MHz} ; \mathrm{CDCl}_{3}\right) 19.7$ and 19.9 (C-8 and C-9), 25.5, 28.0 and 29.7 (C-5, C6 and $\mathrm{C}-3), 30.3\left[\mathrm{C}\left(\mathrm{CH}_{3}\right)\right], 44.0(\mathrm{C}-4), 50.1$ and $50.7(\mathrm{C}-7$ and $\mathrm{C}-1), 54.5\left[\mathrm{C}_{\left.\left(\mathrm{CH}_{3}\right)_{3}\right]} 58.9(\mathrm{C}-10)\right.$, $78.0(\mathrm{C}-2), 128.9\left(\mathrm{C}-2^{\prime}\right), 130.9\left(\mathrm{C}-3^{\prime}\right)$ and $165.9(\mathrm{C}=\mathrm{O})$.

Similar reaction of crude $N, N$-dicyclohexyl-2-exo-hydroxybornane-10-sulfonamide (7j) afforded two products:

Fraction 1. 2-exo-Acryloyloxy- $N, N$-dicyclohexylbornane-10-sulfonamide (9j). (42\%) white solid slightly contaminated with compound $\mathbf{1 1 j} ; \delta_{\mathrm{H}}\left(400 \mathrm{MHz} ; \mathrm{CDCl}_{3}\right) 0.89$ and $1.01(6 \mathrm{H}, 2 \times \mathrm{s}$, 8- and 9-Me), 1.05-3.22 (31H, series of $\mathrm{m}, 13 \times \mathrm{CH}_{2}$ and $\left.3 \times \mathrm{CH}\right), 5.09(1 \mathrm{H}, \mathrm{d}, J 7.6 \mathrm{~Hz}, 2-\mathrm{CH})$, $5.79\left(1 \mathrm{H}, \mathrm{d}, J 10.4 \mathrm{~Hz}, 3^{\prime}-\mathrm{H}_{\mathrm{E}}\right), 6.10\left(1 \mathrm{H}, \mathrm{dd}, J 10.8\right.$ and $\left.13.6 \mathrm{~Hz}, 2^{\prime}-\mathrm{H}\right)$ and $6.35(1 \mathrm{H}, \mathrm{d}, J 17.2 \mathrm{~Hz}$, $\left.3^{\prime}-\mathrm{H}_{\mathrm{Z}}\right) ; \delta_{\mathrm{C}}\left(100 \mathrm{MHz} ; \mathrm{CDCl}_{3}\right) 20.0$ and 20.5 (C-8 and C-9), $44.6(\mathrm{C}-4), 57.4(2 \times \mathrm{CHN}), 78.4(\mathrm{C}-$ 2), 129.2 (C-2'), 129.8 (C-3'), 25.2, 26.4, 32.8 (cyclohexyl $\mathrm{CH}_{2}$ ), 27.0, 29.9, 39.1 and 49.1 (camphor $\left.\mathrm{CH}_{2}\right), 49.5$ and $53.7(\mathrm{C}-1$ and $\mathrm{C}-7)$ and $164.5(\mathrm{C}=\mathrm{O})$.

Fraction 2. 2-exo-[(3-Chloropropanoyl)oxy]- $\mathbf{N}, \mathbf{N}$-dicyclohexylbornane-10-sulfonamide (11j). (58\%), white crystals slightly contaminated with compound $\mathbf{9 j}$; $\delta_{\mathrm{H}}\left(400 \mathrm{MHz} ; \mathrm{CDCl}_{3}\right) 0.88$ and $0.99\left(6 \mathrm{H}, 2 \times \mathrm{s}, 8\right.$ - and 9-Me), 1.05-3.22 $\left(31 \mathrm{H}\right.$, series of $\mathrm{m}, 13 \times \mathrm{CH}_{2}$ and $\left.3 \times \mathrm{CH}\right), 4.99(1 \mathrm{H}, \mathrm{d}, J$ $7.6 \mathrm{~Hz}, 2-\mathrm{CH}), 2.77\left(2 \mathrm{H}, \mathrm{t}, J 7.0 \mathrm{~Hz}, 2^{\prime}-\mathrm{CH}_{2}\right)$ and $3.76\left(2 \mathrm{H}, \mathrm{m}, 3^{\prime}-\mathrm{CH}_{2}\right) ; \delta_{\mathrm{C}}\left(100 \mathrm{MHz} ; \mathrm{CDCl}_{3}\right)$ 20.0 and 20.4 (C-8 and C-9), 57.4 ( $2 \times$ CHN of cyclohexyl), 37.8 (C-2'), 39.1 (C-3'), 44.5 (C-4), $79.2(\mathrm{C}-2), 25.1,26.5$ and 32.8 (cyclohexyl $\left.\mathrm{CH}_{2}\right), 27.0,30.3$, and 49.2 (camphor $\left.\mathrm{CH}_{2}\right), 49.5$ and $53.97(\mathrm{C}-1$ and $\mathrm{C}-7)$ and $168.7(\mathrm{C}=\mathrm{O})$.

Exploratory МВH reactions. General procedure, exemplified by the preparation of the diastereomeric $\mathrm{MBH}$ products 12 and 13. To a solution of 2-exo-acryloyloxy- $N$-benzylbornane10-sulfonamide (9a) $(0.03 \mathrm{~g}, 0.07 \mathrm{mmol})$ in $\mathrm{CDCl}_{3}(0.1 \mathrm{~mL})$ was added pyridine-4-carbaldehyde $(0.01 \mathrm{~g}, 0.07 \mathrm{mmol})$, and DABCO $(0.001 \mathrm{~g}, 0.01 \mathrm{mmol})$. The solution was stirred at r.t. for $90 \mathrm{~h}$ and then concentrated in vасио. The residue was purified by flash chromatography and HPLC [elution with hexane-EtOAc (8: 2)] to afford a mixture of the diastereomeric MBH products $12 \mathrm{a}$ as yellow crystals $\left(0.03 \mathrm{~g}, 91 \%\right.$; 8\% d.e.), m.p. $98-100{ }^{\circ} \mathrm{C}$ (Found: $\mathrm{MH}^{+}, 485.2111 . \mathrm{C}_{26} \mathrm{H}_{33} \mathrm{~N}_{2} \mathrm{O}_{5} \mathrm{~S}$ requires $M+\mathrm{H}: 485.2110) ; v_{\max }(\mathrm{ATR}) / \mathrm{cm}^{-1} 3274(\mathrm{OH}) ; \delta_{\mathrm{H}}\left(400 \mathrm{MHz} ; \mathrm{CDCl}_{3}\right) 0.67(3 \mathrm{H}, \mathrm{s}, 9-\mathrm{Me})$, $0.68(3 \mathrm{H}, \mathrm{s}, 8-\mathrm{Me}), 0.92-1.87\left(7 \mathrm{H}\right.$, series of $\left.\mathrm{m}, \mathrm{H}_{2}-3,5,6, \mathrm{H}-4\right), 2.64$ and $3.19(2 \mathrm{H}, 2 \times \mathrm{d}, 14.0 \mathrm{~Hz}$, 10- $\left.\mathrm{CH}_{2}\right), 4.90\left(2 \mathrm{H}, \mathrm{s}, \mathrm{PhCH}_{2}\right), 4.93(2 \mathrm{H}$, br s, $2-\mathrm{H}$ and $\mathrm{NH}), 5.49(1 \mathrm{H}, \mathrm{s}, \mathrm{CHOH}), 6.12(1 \mathrm{H}$, br s, 
$\mathrm{OH}), 5.72$ and $6.17\left(2 \mathrm{H}, 2 \times \mathrm{s}, 9^{\prime}-\mathrm{CH}_{2}\right), 7.40(2 \mathrm{H}, \mathrm{d}, J 4.5 \mathrm{~Hz}, \mathrm{ArH}), 8.38(5 \mathrm{H}$, overlapping signals, $\mathrm{ArH})$ and $8.56(2 \mathrm{H}, \mathrm{d}, J 4.5 \mathrm{~Hz}, \mathrm{ArH})$.

Similar reactions afforded the following $\mathrm{MBH}$ products as diastereomeric mixtures.

MBH products 13a. Brown oil (0.03 g, 100\%; 33\% d.e.) (Found: $\mathrm{MH}^{+}, 499.2252 . \mathrm{C}_{27} \mathrm{H}_{35} \mathrm{~N}_{2} \mathrm{O}_{5} \mathrm{~S}$ requires, $M+\mathrm{H}: 499.2267) ; v_{\max }(\mathrm{ATR}) / \mathrm{cm}^{-1} 3266(\mathrm{OH}) ; \delta_{\mathrm{H}}\left(400 \mathrm{MHz} ; \mathrm{CDCl}_{3}\right) 0.78(3 \mathrm{H}, \mathrm{s}, 9-\mathrm{Me})$, $0.93(3 \mathrm{H}, \mathrm{s}, 8-\mathrm{Me}), 1.14-1.97\left(6 \mathrm{H}\right.$, series of $\left.\mathrm{m}, \mathrm{H}_{2}-3,5,6\right), 2.17(1 \mathrm{H}, \mathrm{s}, 4-\mathrm{H}), 2.65$ and $3.27(2 \mathrm{H}, 2$ $\left.\times \mathrm{d}, 14.0 \mathrm{~Hz}, 10-\mathrm{CH}_{2}\right), 4.18\left(2 \mathrm{H}, \mathrm{d}, J 5.6 \mathrm{~Hz}, \mathrm{PhCH}_{2}\right), 4.27(1 \mathrm{H}, \mathrm{t}, 4.8 \mathrm{~Hz}, \mathrm{NH}), 5.00(1 \mathrm{H}, \mathrm{d}, J 5.6$ $\mathrm{Hz}, 2-\mathrm{H}), 5.46(1 \mathrm{H}, \mathrm{s}, \mathrm{OH}), 5.64(1 \mathrm{H}, \mathrm{s}, \mathrm{CHOH}), 5.95$ and $6.29\left(2 \mathrm{H}, 2 \times \mathrm{s}, 9^{\prime}-\mathrm{CH}_{2}\right), 7.14(2 \mathrm{H}, \mathrm{m}$, $\mathrm{ArH}), 7.33$ (5H, overlapping signals, $\mathrm{ArH})$ and $7.70(1 \mathrm{H}, \mathrm{m}, \mathrm{ArH})$.

MBH products 12b. Yellow oil (0.03 g, 92\%; 7\% d.e.) (Found: $\mathrm{MH}^{+}, 505.1542 . \mathrm{C}_{25} \mathrm{H}_{30} \mathrm{ClN}_{2} \mathrm{O}_{5} \mathrm{~S}$ requires, $M+\mathrm{H}$ : 505.1564); $v_{\max }(\mathrm{ATR}) / \mathrm{cm}^{-1} 3374(\mathrm{OH}) ; \delta_{\mathrm{H}}\left(400 \mathrm{MHz} ; \mathrm{CDCl}_{3}\right) 0.76(3 \mathrm{H}, \mathrm{s}, 9-\mathrm{Me})$, $0.81(3 \mathrm{H}, \mathrm{s}, 8-\mathrm{Me}), 0.97-1.90\left(7 \mathrm{H}\right.$, series of $\left.\mathrm{m}, \mathrm{H}_{2}-3,5,6, \mathrm{H}-4\right), 3.05$ and $3.44(2 \mathrm{H}, 2 \times \mathrm{d}, J 14.4 \mathrm{~Hz}$, $\left.10-\mathrm{CH}_{2}\right), 5.00(2 \mathrm{H}, \mathrm{m}, 2-\mathrm{H}$ and $\mathrm{NH}), .93(2 \mathrm{H}, \mathrm{br} \mathrm{s}, 2-\mathrm{H}$ and $\mathrm{NH}), 5.53(1 \mathrm{H}, \mathrm{s}, \mathrm{CHOH}), 5.67$ and $6.16\left(2 \mathrm{H}, 2 \times \mathrm{s}, 9^{\prime}-\mathrm{CH}_{2}\right), 6.43(1 \mathrm{H}, \mathrm{br} \mathrm{s}, \mathrm{OH})$ and 6.99-7.31 (8H, overlapping signals, $\left.\mathrm{ArH}\right)$.

MBH products 13b. Yellow oil (0.03 g, 98\%; 21\% d.e.) (Found: $\mathrm{MH}^{+}, 520.3279 . \mathrm{C}_{25} \mathrm{H}_{30} \mathrm{ClN}_{2} \mathrm{O}_{5} \mathrm{~S}$ requires, $M+\mathrm{H}: 519.1720)$; $v_{\max }(\mathrm{ATR}) / \mathrm{cm}^{-1} 3510(\mathrm{OH}) ; \delta_{\mathrm{H}}\left(400 \mathrm{MHz} ; \mathrm{CDCl}_{3}\right) 0.69(3 \mathrm{H}, \mathrm{s}, 9-\mathrm{Me})$, $0.82(3 \mathrm{H}, \mathrm{s}, 8-\mathrm{Me}), 0.99-1.95\left(7 \mathrm{H}\right.$, series of $\left.\mathrm{m}, \mathrm{H}_{2}-3,5,6, \mathrm{H}-4\right), 2.63\left(3 \mathrm{H}, \mathrm{s}, \mathrm{ArCH}_{3}\right), 2.91$ and 3.30 $\left(2 \mathrm{H}, 2 \times \mathrm{d}, 14.4 \mathrm{~Hz}, 10-\mathrm{CH}_{2}\right), 5.05(2 \mathrm{H}, \mathrm{m}, 2-\mathrm{H}$ and $\mathrm{NH}), 5.52(1 \mathrm{H}, \mathrm{s}, \mathrm{CHOH}), 5.64$ and $6.15(2 \mathrm{H}$, $\left.2 \times \mathrm{s}, 9^{\prime}-\mathrm{CH}_{2}\right), 5.71(1 \mathrm{H}$, br s, $\mathrm{OH})$ and 6.97-7.75 (7H, overlapping signals, $\left.\mathrm{ArH}\right)$.

The known MBH products $\mathbf{1 2 f ^ { 6 }}$ as yellow crystals $(0.03 \mathrm{~g}, 93 \% ; 8 \%$ d.e. $)$.

The known MBH products $\mathbf{1 3 f ^ { 6 }}$ as a brown oil ( $0.03 \mathrm{~g}, 96 \% ; 15 \%$ d.e. $)$.

\section{Acknowledgements}

The authors thank the National Research Foundation (NRF) and Rhodes University for generous bursary and financial support. This work is based on research supported by the National Research Foundation, and any opinion, findings and conclusions or recommendations expressed in this material are those of the authors and therefore the NRF does not accept any liability in regard thereto.

\section{References}

1. Money, T. Nat. Prod. Rep. 1985, 2, 253. http://dx.doi.org/10.1039/np9850200253

2. Oppolzer, W. Pure Appl. Chem. 1990, 62, 1241.

3. Helmchen, G.; Wegner, G. Tetrahedron Lett. 1985, $26,6047$. http://dx.doi.org/10.1016/S0040-4039(00)95121-9 
4. Evans, M. D.; Kaye, P. T. Synth. Commun. 1998, 28, 4485.

http://dx.doi.org/10.1080/00397919808004484

5. Kaye, P. T.; Molema, W. E. Chem. Commun. 1998, 2479. http://dx.doi.org/10.1039/a806867d

6. Duggan, A. R.; Kaye, P. T. J. Chem. Res. 2007, 148. http://dx.doi.org/10.3184/030823407X196953

7. Oppolzer, W.; Chapuis, C.; Bernardinelli, G. Tetrahedron Lett. 1984, 25, 5885. http://dx.doi.org/10.1016/S0040-4039(01)81711-1

8. Oppolzer, W.; Marco-Contelles, J. Helv. Chim. Acta 1986, 69, 1699. http://dx.doi.org/10.1002/hlca.19860690725

9. Oppolzer, W.; Dudfield, P. Tetrahedron Lett. 1985, 26, 5037.

10. Oppolzer, W.; Moretti, R. Tetrahedron 1988, 44, 5541. http://dx.doi.org/10.1016/S00404020(01)86059-2

11. Bode, M. L.; Kaye, P. T. Tetrahedron Lett. 1991, 32, 5611. http://dx.doi.org/10.1016/00404039(91)80098-Q

12. Schäfer, A.; Fischer, B.; Paul, H.; Bosshard, R.; Hesse, M.; Viscontinif, M. Helv. Chim. Acta 1992, 75, 1955 .

http://dx.doi.org/10.1002/hlca.19920750621

13. Schäfer, M.; Drayue, M.; Springer, A.; Zacharias, P.; Meerholz, K. Eur. J. Org. Chem. 2007, 5162.

http://dx.doi.org/10.1002/ejoc.200700199

14. 14. Kozakiewicz, A.; Ullrich, M.; Welniak, M.; Wojtczak, A. J. Mol. Catal. A: Chem. 2010, $326,128$. http://dx.doi.org/10.1016/j.molcata.2010.04.019

15. Lewis, F. W.; McCabe, T. C.; Grayson, D. H. Tetrahedron 2011, 67, 7517. http://dx.doi.org/10.1016/j.tet.2011.07.081

16. Shubber, A. K.; Kazandji, S. Y. Iraqi J. Sci. 1990, 31, 529.

17. Ramon, D. J.; Yus, M. Tetrahedron: Asymmetry 1997, 8, 2479. http://dx.doi.org/10.1016/S0957-4166(97)00272-3 